\title{
MANIPULACIÓN REMOTA EN INSTALACIONES CON ELEVADO GRADO DE RADIACIÓN
}

\author{
Sofía Coloma, Jose M. Breñosa, Luís E. Rubio, Manuel Ferre y Rafael Aracil
}

\author{
Centro de Automática y Robótica (CAR) UPM-CSIC, Madrid, España. \\ email:\{sofia.coloma@upm.es, jose.brenosa@upm.es, luiseduardo.rubio@upm.es,m.ferre@upm.es, \\ rafael.aracil@upm.es \}
}

\begin{abstract}
Resumen
La problemática principal en las instalaciones enfocadas a la fusión nuclear, radica en las condiciones ambientales tan extremas que se encuentran en el interior de las áreas de operación y la necesidad de que los equipos cumplan requisitos muy restrictivos para poder aguantar altos niveles de radiación, magnetismo, ultravacio, temperatura, etc. Como no es viable la ejecución de tareas directamente por parte de humanos, se deben utilizar dispositivos de manipulación remota para llevar a cabo los procesos de operación y mantenimiento. Sin embargo, las tareas de teleoperación en entornos con radiación, continúan siendo un reto tecnológico debido al nivel de complejidad y los problemas que presentan. En este sentido, se proponen diferentes soluciones para abordar los inconvenientes del mantenimiento en instalaciones con altos niveles de radiación y poder así garantizar un correcto funcionamiento. Las soluciones expuestas están basadas en la utilización de un multiplexor para la transmisión de señales y la utilización de un algoritmo de control para mejorar la teleoperación en estos entornos, las cuales han sido evaluadas previamente, y han ofreciendo mejoras con respecto a tecnologías actuales.
\end{abstract}

Palabras Clave: manipulación remota, radiación, telerrobótica, escalado, rate-position, multiplexor, radhard.

\section{INTRODUCCIÓN}

A día de hoy, las investigaciones y estudios en el sector nuclear continúan en auge en busca de mejoras y soluciones aplicables a casos prácticos. Por ello, multitud de instalaciones a nivel mundial están destinadas a la investigación en entornos irradiados.
Este artículo hace hincapié en las instalaciones enfocadas a la energía nuclear para el estudio de la generación de electricidad mediante fusión nuclear, siendo citadas: JET (Joint European Torus) [13], ITER (International Thermonuclear Experimental Reactor) [14], IFMIF (International Fusion Materials Irradiation Facility) [5] y DONES (DEMO-Oriented Neutron Source) [7]. Estas instalaciones junto con otras, deben operar bajo ciertos niveles de radiación para culminar con el trabajo para el cual han sido diseñadas. Ahora bien, la radiación esta contenida en recintos controlados y por ende, la radiación no debe suponer un riesgo para el ser humano ni para el entorno fuera del área de trabajo.

A diferencia de otro tipo de instalaciones, la radiación provoca mayor deterioro físico-químico en los materiales ubicados en este tipo de entornos. Por este motivo, se debe procurar seleccionar materiales adecuados a este tipo de condiciones (RadHard) [4], blindar la sala de operaciones y proteger en la medida de lo posible, la electrónica expuesta a la radiación con el objetivo de evitar fallos indeseados. Sin embargo, para garantizar mayor fiabilidad y disponibilidad, también es necesario realizar paradas de mantenimiento programadas cada cierto periodo de actividad [7]. Realizándose siempre que sea posible, operaciones de mantenimiento mediante manipulación remota de robots para poder acceder a las zonas críticas de la instalación [10,11]. De este modo, se evita la exposición del ser humano ante este tipo de entornos y largas esperas hasta que los niveles de radiación bajen a dosis permitidas por el ser humano.

A pesar de las grandes ventajas que supone la manipulación remota de equipos y robots bajo estas condiciones, hay una serie de retos (radiación, dimensiones, precisión y pesos) que se deben abordar en estos casos para el correcto funcionamiento del sistema. Ya que, éstos pueden suponer un peligro para la salud del ser humano, periodos de inactividad 
indeseados y un coste elevado para la propia instalación por cada día de inactividad en la planta. Por este motivo, a lo largo de este artículo se proponen una serie de técnicas que permitan atenuar los problemas originados en instalaciones con un entorno irradiado.

\section{RETOS DE LA TELEOPERACIÓN EN ENTORNOS CON RADIACIÓN}

Debido a los altos niveles de radiación después del periodo de funcionamiento en el área de trabajo, lo apropiado es que las tareas de mantenimiento se realicen con robots o maquinaría controlada mediante manipulación remota. Para ello, los componentes que permiten realizar las tareas de mantenimiento, como grúas, servo-manipuladores o pinzas de agarre se localizan en el área de trabajo. Éstas son teleoperadas a distancia mediante una interfaz controlada por el operador ubicado en una sala de control, la cual es un área totalmente segura sin el riesgo de exposición del trabajador a entornos irradiados.

Las tareas de telemanipulación en grandes instalaciones científicas con radiación son un reto tecnológico en el que muchas son las investigaciones que tratan de resolver los problemas que se derivan de las condiciones ambientales del entorno $[6,8,12]$.

Para tener una estimación del escenario de operación en este tipo de entornos, la tabla 1 muestra el tipo de

Tabla 1 - Datos estimados de inspecciones en ITER

\begin{tabular}{|c|c|c|c|}
\hline & Inspección programada & $\begin{array}{l}\text { Inspección } \\
\text { No programada }\end{array}$ & $\begin{array}{l}\text { Mantenimiento } \\
\text { de maquinaria }\end{array}$ \\
\hline Escenario listo para operar & $\begin{array}{l}1 \text { día después de } \\
\text { operaciones de plasma }\end{array}$ & $\begin{array}{ll}1 \text { hora después } & \text { de } \\
\text { operaciones } & \text { de } \\
\text { plasma } & \end{array}$ & $\begin{array}{l}2 \text { semanas después de } \\
\text { operaciones de plasma }\end{array}$ \\
\hline Atmósfera & \multicolumn{2}{|c|}{ UHV condiciones $\left(>10^{\wedge} 5 \mathrm{~Pa}\right)$} & Aire $\operatorname{seco}(\approx 1$ bar $)$ \\
\hline Campo magnético (generado) & \multicolumn{2}{|l|}{$5-8$ Tesla } & Aprox. $\approx 0$ Tesla \\
\hline $\begin{array}{ll}\text { Campo } & \text { magnético } \\
\text { (almacenado) } & \end{array}$ & \multicolumn{2}{|l|}{0.3 Tesla } & Aprox. $\approx 0$ Tesla \\
\hline Radiación (generada) & $1,500 \mathrm{~Gy} / \mathrm{h}$ & $15,000 \mathrm{~Gy} / \mathrm{h}$ & $300 \mathrm{~Gy} / \mathrm{h}$ \\
\hline Radiación (almacenada) & \multicolumn{3}{|c|}{$\begin{array}{l}\text { Flujo de Neutrones total estimado (BPP) } 5.05 \mathrm{E} 13 \mathrm{n} / \mathrm{cm} 2 \\
\text { Dosis Gamma estimada (BPP) } 1.41 \mathrm{E}+02 \text { Gy } \\
\text { Radiación adicional del agua de refrigeración } 16 \mathrm{~N} \text { (TBD) }\end{array}$} \\
\hline $\begin{array}{lll}\text { Duración } & \text { estimada } & \text { de } \\
\text { Inspección } & & \end{array}$ & $\begin{array}{l}12 \mathrm{~h} / \mathrm{sem}, \text { dos veces al } \\
\text { mes, durante } 7,5 \text { años }\end{array}$ & $\begin{array}{l}12 \mathrm{~h} / \mathrm{sem} \text {, dos veces al } \\
\text { mes, durante } 7,5 \text { años }\end{array}$ & $\begin{array}{l}\text { 60h/mes, durante } 32 \\
\text { meses }\end{array}$ \\
\hline $\begin{array}{l}\text { Dosis Total de Gamma } \\
\text { Dosis Total de Neutrones }\end{array}$ & $\begin{array}{l}2.7 \mathrm{MGy} \\
5.07 \mathrm{E} 13 \mathrm{n} / \mathrm{cm} 2\end{array}$ & $\begin{array}{l}27 \mathrm{MGy} \\
5.07 \mathrm{E} 13 \mathrm{n} / \mathrm{cm} 2\end{array}$ & $\begin{array}{l}0.6 \mathrm{MGy} \\
5.07 \mathrm{E} 13 \mathrm{n} / \mathrm{cm} 2\end{array}$ \\
\hline
\end{tabular}

mantenimiento en función de las condiciones de operaciones en la instalación de ITER.

\subsection{PROBLEMÁTICA DEL ENTORNO REMOTO}

Las instalaciones científicas cuyas investigaciones implican tratar con materiales expuestos a la radioactividad, como las anteriormente citadas $[5,7,13,14]$ requieren de servicios de manipulación remota para resolver mayoritariamente las siguientes necesidades:

\section{- Radiación del entorno}

La fusión nuclear genera una radiación que es dañina para el entorno donde es contenida, ya que los componentes se degradan con mayor celeridad, las señales de comunicación se ven afectadas, la electrónica se deteriora y la exposición humana a ciertos niveles resulta peligrosa e inviable. Esto justifica la utilización de la telerrobótica y dispositivos operados por control remoto en este tipo de entornos.

\section{- Grandes dimensiones del espacio de trabajo}

En dichas instalaciones el operador utiliza un dispositivo maestro para poder enviar órdenes de movimiento al robot esclavo o a la maquinaria ubicada en el entorno remoto de grandes dimensiones donde se realizan las operaciones de mantenimiento [3]. La diferencia de dimensiones implica que se utilicen técnicas de escalado con una consecuente pérdida de resolución o el uso constante de modos de control maestro-esclavo para poder abarcar toda el área del entorno remoto. 


\begin{abstract}
- Manipulación de cargas elevadas
En las tareas de telemanipulación se deberá conseguir la mayor inmersión posible en el entorno con el objetivo de facilitar el control de las acciones remotas que debe realizar el operador. Por este motivo, es necesaria la utilización de dispositivos maestros hápticos para que el operador al manipularlo perciba las fuerzas de la interacción del robot esclavo en el entorno [9]. Permitiendo tener mayor percepción tanto en la manipulación como en las fuerzas generadas en el entorno, tomando consciencia ante colisiones o esfuerzos inadecuados. Esto es muy relevante en la telemanipulación de cargas elevadas, ya que una mala gestión de la reflexión de fuerzas puede producir daños en el entorno remoto o lesiones en el operador.
\end{abstract}

\section{- Realización de tareas con alta precisión}

El objetivo de todas las tareas de telemanipulación es poder realizarla con la mayor precisión posible, ya que por lo general, éstas son tareas delicadas en cuanto a que no se pueden producir daños en el entorno remoto, por lo que el margen de error debe ser mínimo.

Este tipo de tareas continúa siendo un reto tecnológico para la telemanipulación, debido a la cantidad de inconvenientes que suponen las comunicaciones y los componentes electrónicos localizados en entornos con altos niveles de radiación.

\section{REDUCCIÓN EN LA TRANSMISIÓN DE SEÑALES: MULTIPLEXOR}

Debido a las altas dosis de radiación y a lo que sufre la electrónica ante este tipo de condiciones ambientales, es necesario que estos elementos sean blindados para protegerlos del bombardeo de neutrones y partículas. Éstos tienen que ser reemplazados periódicamente para evitar que lleguen al final de su vida útil. Una posible alternativa consiste en la deslocalización de la electrónica situándola en un lugar libre de radiación, pero esto conlleva a otros problemas asociados al volumen de cableado y de ruido en todas las señales.

Una solución ante esta problemática es situar una electrónica genérica RadHard de tipo multiplexor que digitalice las señales cerca de la fuente y que transmita varias señales por un único cable hasta la sala de control donde se demultiplexe en su electrónica específica como se muestra en la figura 1. Así, se solucionarían los problemas de blindaje de equipos electrónicos, ruido en las señales y volumen de cableado.
Se deberá entonces realizar un estudio teórico sobre la viabilidad del desarrollo de una electrónica multiplexora, que resista ante condiciones extremas de radiación con niveles en torno a las operaciones de mantenimiento de las instalaciones como ITER o DONES.

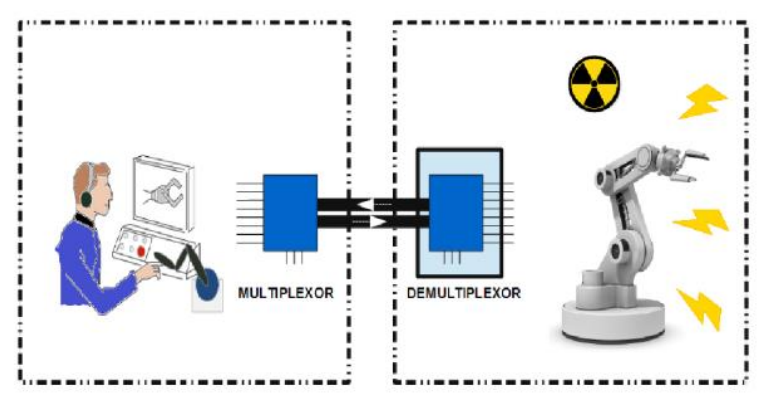

Figura 1: Esquema básico de la comunicación con multiplexado de señales.

\section{ADAPTACIÓN DE LOS ESPACIOS DE TRABAJO CON "RATE - POSITION CONTROLLER"}

En el caso de guiar un robot a distancia en grandes espacios de trabajo, generalmente se utilizan dos modos de control: posición y velocidad $[2,15]$. Utilizar la combinación de ambos controles es mejor cuando los espacios de trabajo del dispositivo maestro y el dispositivo esclavo son diferentes.

Habitualmente en estos casos, se indexa el espacio de trabajo del maestro haciendo una desvinculación cuando el dispositivo maestro alcanza su límite mecánico, así éste se traslada a una nueva posición que permita continuar con el proceso, cambiando de modo de control. No obstante, este método de indexación genera desorientación por los cambios en la referencia de la posición. Por esta razón, se propone un método más intuitivo, el rate-position control [1], el cual permite cambiar de modo de control de forma más natural y ayuda a paliar problemas en el campo de la telerrobótica. Es por ello que, se ha decidido utilizar este algoritmo para solventar los problemas que aparecen en entornos de radiación debido a las grandes dimensiones sobre las que se trabaja.

El rate-positon control divide en áreas esféricas el espacio de trabajo del dispositivo maestro como se muestra en la figura 2, donde se observan los diferentes estados. 


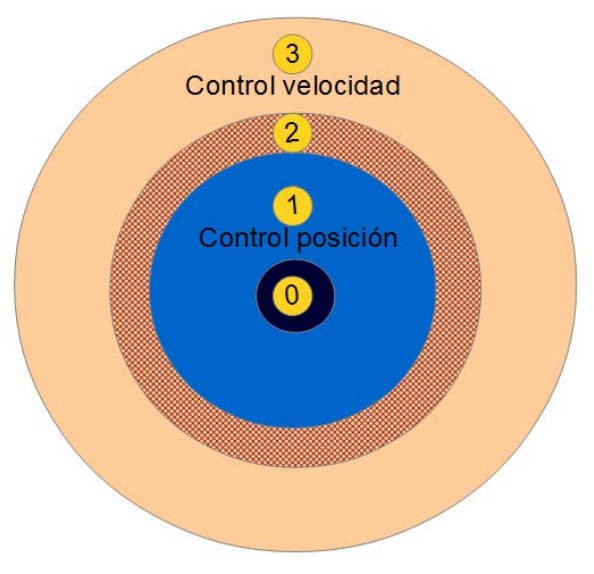

Figura 2: Área del control velocidad-posición.

Estado 0 "ir al centro": al entrar por primera vez en el modo posición, ya sea al inicializarlo, al pasar de control velocidad a posición o por seguridad en una colisión, una fuerza lleva a la posición central (estado 0) al dispositivo maestro con el fin de ubicarlo en el punto central de referencia y sincronizarlo con la posición actual del esclavo.

Estado 1 "control en posición": permite telemanipular al robot esclavo en posición y recibir las fuerzas de interacción con el medio ambiente. El esclavo se limita a imitar los movimientos ejecutados por el maestro, siempre que el dispositivo esté dentro de las dimensiones de la circunferencia del control de posición.

Estado 2 "fase vibratoria": al sobrepasar la zona esférica del control de posición, se entra en el estado 2 , el cual indica al usuario mediante un estímulo vibratorio que ha sobrepasado la posición de espacio de trabajo y se va a producir la transición al control en velocidad.

Estado 3 "control en velocidad": permite teleoperar al esclavo con comandos de velocidad. Además, por seguridad, el operario siente una fuerza proporcional a la distancia hacia al centro, por tanto cuanto más se aleja de la esfera, mayor es la fuerza.

\section{EVALUACIONES}

Expuestas anteriormente diferentes soluciones a los problemas mentados que se pueden producir en tareas de telemanipulación, esta sección quiere profundizar en el estudio de viabilidad de un multiplexor RadHard con el objetivo de mostrar soluciones a los problemas de comunicación y mantenimiento. Posteriormente se explicará la técnica para trabajar en grandes espacios de trabajo.

\subsection{Viabilidad del multiplexor}

En este tipo de instalaciones la conexión entre los equipos remotos y la sala de control es crucial para la consecución de las tareas. Por tanto la fiabilidad de la conexión con toda la instrumentación es un requisito indispensable.

Por lo general, para las comunicaciones se necesita un gran volumen de cableado para abarcar todos los sensores y actuadores de los distintos dispositivos ubicados en el entorno remoto. Suponiendo esto un gran problema para la instalación debido al tipo de entorno, ya que los cables se deterioraran con mayor frecuencia y deberán ser remplazados periódicamente. Por tanto, si el número de cables es elevado, aumentará proporcionalmente el coste a invertir, el tiempo y la dificultar a la hora de realizar la tarea de mantenimiento. Para afrontar dicha tesitura, se puede optar por dos soluciones: la multiplexación o las redes inalámbricas. Sin embargo, si se quiere una mayor fiabilidad se debe optar por la multiplexación.

La idea principal de la tecnología de multiplexado se basa en codificar muchas señales a través de un multiplexor y extraer un único canal compartido por cable en el entorno irradiado. Posteriormente, cuando el cable esté en una zona segura de radiación, un demultiplexor permitirá decodificar la señal y se obtendrá todas esas señales comprimidas, las cuales corresponderán a los datos enviados por los sensores y actuadores del robot. Esto permite reducir la sección y el peso de la cantidad de cableado, ya que varias señales comparten un solo conductor, tal como un cable de cobre o cable de fibra óptica.

Para el correcto funcionamiento y vida útil de la electrónica del multiplexor que se ubica en la zona irradiada, se deben tener una serie de consideraciones:

- Dispositivo RadHard altamente testado y cualificado para la vida útil del multiplexor.

- Blindaje de la electrónica con materiales que apantallen los rayos gamma como el plomo $(\mathrm{Pb})$ o el tungsteno $(\mathrm{W})$.

- Refrigeración para evitar altas temperaturas que afecten a la electrónica.

\subsection{Viabilidad del " rate-position controller"}

Para justificar la validez del algoritmo de control propuesto como solución, es necesario la comparación con los métodos actuales del mercado y la verificación de que aporta una mejora con respecto a sus competidores. Para esta validación se ha propuesto la realización de un experimento de 
telemanipulación que evalúa los distintos algoritmos en una misma tarea.

\subsubsection{Experimento: Telemanipulación de un objeto frágil}

En la validación del rate-position se ha propuesto un experimento que compare dicho método con respecto al método de indexado comentado previamente, y también con el control en posición realizado con un maestro de cinemática similar a la del esclavo.

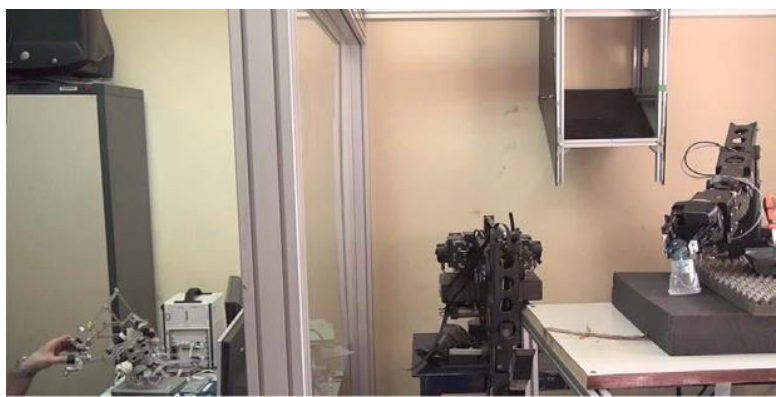

Figura 3: Usuario realizando una telemanipulación compleja.

Para la realización del experimento se utilizarán un brazo robótico hidráulico de 6 grados de libertad de Kraft Telerobotics con una mano robótica de 3 dedos de Robotiq en la parte del esclavo, mientras que en la parte maestra se utilizará un dispositivo háptico de tres dedos con reflexión de fuerzas (3FingerHaptic Device o 3F-HD) desarrollado en la UPM con los métodos de indexado, rate-position, y una combinación entre una interfaz gráfica de la mano y el maestro proporcionado por el fabricante para el control en posición con cinemática similar. El resultado de la combinación de todos los dispositivos se puede observar en la figura 3.

Estos tres métodos serán comparados mediante la realización de una misma tarea, la telemanipulación de un objeto frágil (una bombilla) en entornos no estructurados. Los pasos de la tarea son:

1. La tarea comienza con el robot en una posición indicada (A un metro del objetivo).

2. El usuario tiene que aproximarse al área de manipulación donde la bombilla está localizada encima de un vaso y posicionar el robot en postura de pre-agarre.

3. El usuario controlará la mano robótica para realizar el agarre de la bombilla.

4. El usuario volverá a la posición inicial sin romper o dañar la bombilla.
5. El usuario moverá al robot a la zona de manipulación dónde lo colocará en una posición previa a la apertura de la mano.

6. El usuario controlará la mano robótica para colocar la bombilla en su posición final.

El experimento fue realizado 30 veces por dos sujetos con cada uno de los tres métodos. La tarea procesada por los sujetos consistía en realizar acciones de manipulación remota con un robot que incluía en el extremo una mano robótica delicada. Suponiendo que, un error por parte del operador podría romper la mano, por ello, no se permitió llevar a cabo este procedimiento experimental con personas ajenas al grupo de telerrobótica de la UPM. Dichos usuarios tenían experiencia previa con el dispositivo háptico 3-Finger $\mathrm{y}$ con los controladores del fabricante. Sin embargo, ellos no estaban familiarizados con las técnicas de indexación o el rate-position controller.

\section{- Tiempo para completar la tarea}

Como se puede apreciar en la figura 4, el tiempo para realizar la manipulación compleja de un objeto frágil es, para el controlador en rate-position, 68.9 segundos; mientras que para el indexado la media de tiempo son 75.33 segundos. Usando el maestro de cinemática similar se obtiene un tiempo de media de 70.67 segundos.

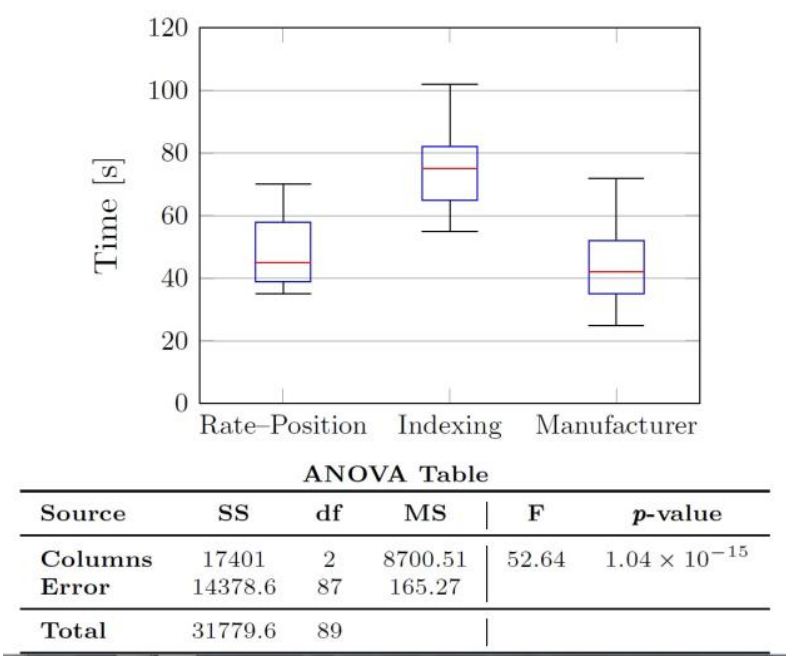

Figura 4: ANOVA: Comparación de tiempos cuando se realiza una tarea de manipulación con destreza con diferentes métodos de control.

El tiempo entre el rate-position y el maestro de cinemática similar son parecidos, mientras que el indexado presenta un valor medio mucho mayor. Esto puede ser debido a las características del indexado en las cuales el operador tiene que volver una y otra vez a las mismas posiciones para cubrir 
distancias largas, lo cual provoca cansancio al operador.

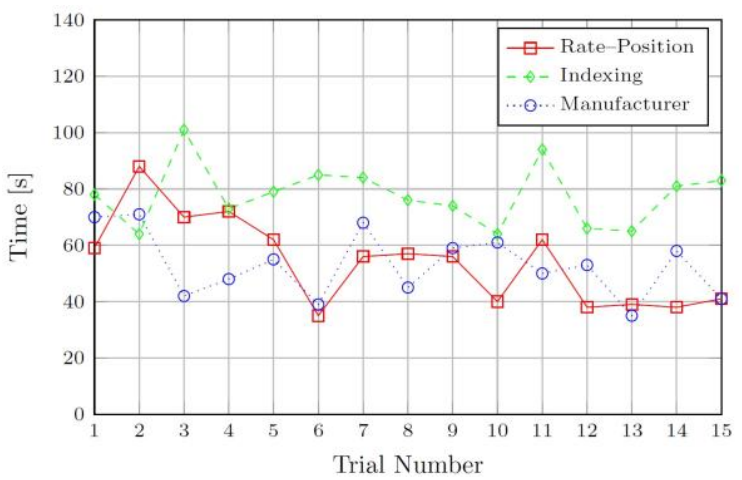

Figura 5: Tiempo de entrenamiento al realizar una tarea de manipulación diestra con diferentes métodos de control.

La figura 5 muestra que, el tiempo de entrenamiento para el rate-position controller, se reduce en media de 70 a 45 segundos después de cinco ensayos, en cambio el modo de indexación se mantiene constante aproximadamente en 80 segundos (no se reduce durante los 15 ensayos) y para el controlador del fabricante, el tiempo se reduce de 65 a 45 segundos después de la tercera prueba. Esto muestra que cinco ensayos son suficientes para acostumbrarse al método rate-position controller y realizar una tarea diestra. En este caso, el control por indexado no presenta una reducción de tiempo durante los ensayos, lo cual es admisible, ya que por lo general los sujetos son entrenados para usar una interfaz de ordenador con un modo de control basado en indexación, como un ratón de ordenador. Este control parecía muy intuitivo para mover el robot, sin embargo, el mayor valor de tiempo se debe a que se debían mover más lejos con el dispositivo esclavo que con el dispositivo maestro, además de mantener un botón pulsado con el fin de desacoplar los dos sistemas.

\section{- Tasa de éxito de la tarea}

En la figura 6, se puede ver que mientras el rateposition y el indexado han alcanzado una tasa del $100 \%$ de éxito, en el controlador del fabricante este porcentaje baja hasta el $93.33 \%$. Después de preguntar a los operadores, ellos mencionan que la principal dificultad cuando usaban el controlador del fabricante fue la coordinación entre los dedos cuando cerraban la mano con la interfaz gráfica mientras mantenían la posición del brazo con el maestro del fabricante. $93.33 \%$ puede parecer una tasa alta de éxito, pero trabajar en un entorno nuclear implica una gran cantidad de riesgo, una tasa de fallo del $6.67 \%$ puede no ser admisible.

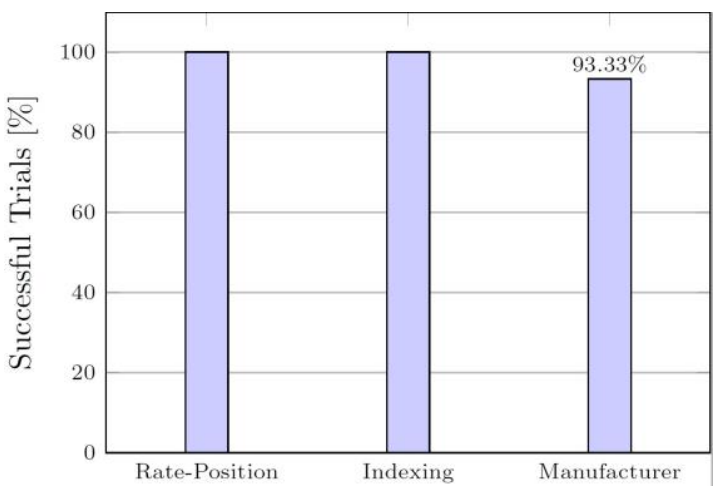

Figura 6: Manipulaciones con éxito cuando se realiza una manipulación con destreza con diferentes métodos de control.

\section{DISCUSION DE RESULTADOS}

Si abordamos una por una las problemáticas de la sección anterior con una combinación de dichas teorías se podrían resolver:

\section{- Radiación del entorno}

La deslocalización de la electrónica mediante un multiplexor RadHard, permite que la radiación no afecte a estos elementos que son los que más sufren. Pese a contar con un Multiplexor RadHard, deberá ser blindado para aportar fiabilidad ante grandes dosis de radiación como las que se encontrarán en DONES o ITER. Las operaciones de mantenimiento de cableado se verán reducidas drásticamente puesto que el multiplexor ayudará a reducir el volumen de éste. Esto también influye positivamente en la maniobrabilidad de dispositivos de manipulación remota puesto que el cable umbilical que les mantiene conectados está reducido en volumen y peso, con lo que la rigidez y solicitaciones que tenga serán menores.

\section{- Grandes dimensiones de trabajo}

Para que el robot pueda sobrepasar los límites del espacio de trabajo impuesto por las limitaciones del dispositivo maestro, el rate-position controller se alterna de forma natural entre dos modos de control que permiten aprovechar todo el espacio de trabajo de los dispositivos maestro y esclavo, sin la pérdida de resolución que conlleva un escalado.

\section{- Manipulación de cargas elevadas}

El rate-position permite reflejar de forma intuitiva las fuerzas ejercidas durante la manipulación al operador. Estas fuerzas pueden ser escaladas para que en caso de tener que trabajar con cargas elevadas o un tiempo prolongado, el operador no se sienta incómodo, agotado o se lesione. Por su parte, el multiplexor realiza una conversión analógico-digital cerca de la fuente, ésto permite que no haya una 
alteración por ruido de los datos transmitidos a través del cableado.

\section{- Realización de algunas tareas con alta precisión La implementación del rate-position como algoritmo de control robusto del sistema maestro-esclavo permite que no haya repercusión negativa a la precisión con temblores, inestabilidades o retrasos. Una implementación de escalado apropiado según la aplicación en el modo de control de posición, permite tener un sistema muy preciso en sistemas con diferencias dimensionales entre maestro y esclavo, dependiendo en última instancia de la precisión del esclavo, que puede ser corregida por el operador que se encuentra en el lazo de control.}

\section{Agradecimientos}

Este trabajo ha sido parcialmente financiado por los proyectos: "TeleScale" (DPI2012-32509) del "Ministerio de Economía y Competividad" y "WPENS/DONES" de EuroFusion.

\section{Referencias}

[1] Barrio, J., Ferre, M., Suárez-Ruiz, F., \& Aracil, R. (2014). A remote handling rate-position controller for telemanipulating in a large workspace. Fusion Engineering and Design, 89(1), 25-28.

[2] Ben-Porat, O., Shoham, M., \& Meyer, J. (2000) Control Design and Task Performance in Endoscopic Teleoperation, Presence: Teleoperators and Virtual Environments, 9(3), 256-267.

[3] Breñosa Martínez, J. M. (2016). Desarrollo de tecnologías de telemanipulación con alto grado de escalado orientadas a la interacción hombre-robot en entornos nucleares (Doctoral dissertation, Industriales).

[4] Claeys, C., \& Simoen, E. (2013). Radiation effects in advanced semiconductor materials and devices (Vol. 57). Springer Science \& Business Media.

[5] Garin, P., \& Sugimoto, M. (2009). Main baseline of IFMIF/EVEDA project.Fusion Engineering and Design, 84(2), 259-264.

[6] Ibarra, A., Perlado, M., Aracil, R., Blanco, D., Ferre, M., García-Cortés, I., ... \& MartinezAlbertos, J. L. (2010). TechnoFusión, a relevant facility for fusion technologies: The remote handling area. Fusion Engineering and Design, 85(7), 1659-1663.
[7] Ibarra.A \& Roman.A (2014) DONES conceptual design report.

[8] Madzharov, V., Mittwollen, M., Leichtle, D., \& njiHermon, G. (2015). Development of a zonal applicability tool for remote handling equipment in DEMO. Fusion Engineering and Design, 98, 1543-1547.

[9] Massie, T. H., \& Salisbury, J. K. (1994, November). The phantom haptic interface: A device for probing virtual objects. In Proceedings of the ASME winter annual meeting, symposium on haptic interfaces for virtual environment and teleoperator systems (Vol. 55, No. 1, pp. 295-300).

[10] Mittwollen, M., Kubaschewski, M., Madzharov, V., Eilert, D., Tian, K., Arbeiter, F., \& Heinzel, V. (2013). Maintenance inside IFMIF Test Facility-(Technical)logistics. Fusion Engineering and Design, 88(9), 2621-2626.

[11] Gómez, J. M., Ollero, A., \& García, A. J. (2006). Teleoperación y telerrobótica. Editorial Pearson Prentice Education. ISBN, 84 -8322.

[12] David, O., Miccichè, G., Ibarra, A., Friconneau, J. P., \& Piazza, G. (2009). Overview of the preliminary remote handling handbook for IFMIF. Fusion Engineering and Design, 84(2), 660-664.

[13] Rebut, P. H., Bickerton, R. J., \& Keen, B. E. (1985). The Joint European Torus: installation, first results and prospects. Nuclear fusion, 25(9), 1011.

[14] Rebut, P. H., Boucher, D., Gambier, D. J., Keen, B. E., \& Watkins, M. L. (1993). The ITER challenge. Fusion engineering and design, 22(1), 7-18.

[15] A. Kazi, Operator Performance in Surgical Telemanipulation, Presence: Teleoperators and Virtual Environments 10 (5) (2001) 495-510. 